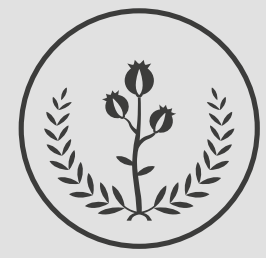

FUCS
Repr per

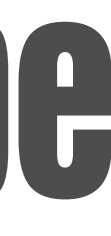

\title{
Vacunas asignadas y aplicadas contra COVID-19 por entidad territorial en Colombia
}

\section{Allocated and applied vaccines against COVID-19 by territorial entity in Colombia}

a Ingeniero. Magister en Gestión de la Tecnología Educativa, Especialista en Administración de la Informática Educativa.

Docente de matemáticas e Investigador, Secretaría de Educación de Soacha, Cundinamarca.

\section{R E S U M E N}

Introducción: para lograr la finalización de esta pandemia, una gran parte del mundo debe ser inmune al virus. La evidencia más segura de lograrlo es una vacuna. Objetivo: mostrar el avance de las dosis asignadas y aplicadas en la vacunación contra SARS-COV-2 en las entidades territoriales de Colombia. Metodología: la presente investigación se realizó bajo un estudio transversal, como fuente de información se obtuvo del plan nacional de vacunación contra el COVID-19 de la página web del Ministerio de Salud y Protección Social, en el período comprendido entre el 17 de febrero y 31 de julio 2021. Resultados: las entidades territoriales con mayor porcentaje de dosis asignadas fueron: Bogotá (18.61), Antioquia (14,92), Valle del Cauca $(8,82)$, Cundinamarca $(6,03)$ y Santander $(5,07)$ y con mayor número de porcentaje de dosis aplicadas de vacunación fueron: Boyacá $(92,85)$, Buenaventura $(92,82)$, Amazonas $(89,03)$, Cundinamarca $(88,22)$, y Nariño (87). Conclusión: es relevante continuar aumentado la asignación de dosis contra SARS-COV-2 en los diferentes entes territoriales de Colombia y a su vez que cada uno aumente su capacidad de aplicaciones de dosis de la vacuna y en especial en lo relacionado con los esquemas completos que permitan llegar a corto plazo a alcanzar la inmunidad de rebaño en el país.

Palabras clave: COVID-19, SARS-COV-2, pandemia, vacunas.

(C) 2021 Fundación Universitaria de Ciencias de la Salud - FUCS. Este es un artículo Open Access bajo la licencia CC BY-NC-ND (http://creativecommons.org/licenses/by-nc-nd/4.0/).

INFORMACIÓN DEL ARTÍCULO

Historia del artículo:

Fecha recibido: agosto 5 de 2021 Fecha aceptado: septiembre 8 de 2021

\footnotetext{
Autor para correspondencia.

Jorge Enrique Díaz Pinzón jediazp@unal.edu.co
}

DOI

10.31260/RepertMedCir.01217372.1266 


\section{A B S T R AC T}

Introduction: to achieve the end of this pandemic, a vast majority of people of the world must be immune to the virus. The safest evidenced way of achieving this is through a vaccine. Objective: to show the progress of the doses assigned and applied during vaccination against SARS-COV-2 in the territorial entities of Colombia. Methodology: a cross-sectional study was used for this research. Data was obtained from the Ministry of Health and Social Protection website based on the national vaccination plan against COVID-19 in the period from February 17 to July 31 2021. Results: the territorial entities with the highest percentage of assigned doses were: Bogota (18.61), Antioquia (14.92), Valle del Cauca (8.82), Cundinamarca (6.03) and Santander (5. 07) and with the highest percentage of applied vaccination doses were: Boyaca (92.85), Buenaventura (92.82), Amazonas (89.03), Cundinamarca (88.22), and Nariño (87). Conclusion: it is relevant to continue increasing the allocation of doses of vaccines against SARS-COV-2 and the vaccine application capacity in the different territorial entities of Colombia, underlining the importance of completing the recommended vaccination courses, enabling the achievement of herd immunity in the country in the short term.

Key words: COVID-19, SARS-COV-2, pandemic, vaccines.

(C) 2021 Fundación Universitaria de Ciencias de la Salud - FUCS.

This is an open access article under the CC BY-NC-ND license (http://creativecommons.org/licenses/by-nc-nd/4.0/).

INTRODUCCIÓN

Para poner fin a esta pandemia, una gran parte del mundo debe ser inmune al virus. La evidencia más segura de lograrlo es con una vacuna, tecnología en la que la humanidad ha confiado a menudo en el pasado para reducir el número de muertes por enfermedades infecciosas. ${ }^{1}$ El camino para lograr vacunas seguras y efectivas es primordial para poner fin a la pandemia de COVID-19, por lo que es muy tranquilizador ver tantas vacunas probando y en desarrollo. La OMS trabaja de manera incansable con sus socios para desarrollar, fabricar y distribuir vacunas seguras y eficaces. ${ }^{2}$ A nivel global hay en desarrollo un total de 25 vacunas contra el COVID-19. ${ }^{3}$ Con millones de personas vacunadas se puede indicar con certeza que son seguras y los efectos adversos severos, como reacciones de anafilaxia, se consideran muy infrecuentes. ${ }^{4}$ El virus SARS-COV-2 ha presentado mutaciones o modificaciones en el ARN viral que cambian aminoácidos en ciertas proteínas virales. ${ }^{5}$

Estas variantes podrían hacer menos efectivos los anticuerpos inducidos por las vacunas. En respuesta a esta situación ya hay compañías laborando en vacunas para las variantes del COVID-19. Cualquier problema que surja con una vacuna produciría una mayor impaciencia, pero hay que recordar que no existe un sustituto mejor para certificar la seguridad que los ensayos clínicos bien trazados, doble ciego, prospectivos y aleatorizados. ${ }^{6}$ Dada la gravedad de la pandemia, la autorización pronta de alguna vacuna es contemplada con preocupación por las autoridades sanitarias de todo el mundo. ${ }^{7-11}$ El objetivo de esta investigación es mostrar el avance de las dosis asignadas y aplicadas para la vacunación contra SARS-COV-2 en las entidades territoriales de Colombia, en el periodo comprendido entre el 17 de febrero y el 31 de julio 2021.

\section{METODOLOGÍA}

La presente investigación es un estudio transversal, la fuente de información fue el plan nacional de vacunación contra el COVID-19 de la página web del Ministerio de Salud y Protección Social ${ }^{12}$, entre el periodo comprendido entre el 17 de febrero 2021 a 31 de julio 2021. Además, se manejaron medios matemáticos y estadísticos cotidianos para valorar los resultados de modo concluyente. ${ }^{13-15}$

\section{RESULTA D OS}

En la figura 1 podemos apreciar los valores de las dosis asignadas y aplicadas para la vacunación contra SARSCOV-2 en las entidades territoriales de Colombia, a 31 de julio 2021. En ella se puede inferir que las entidades territoriales con mayor número de dosis asignadas y aplicadas para la vacunación son: Bogotá (5'992.380 y 4'966.730), Antioquia $\left(4^{\prime} 802.193\right.$ y $\left.4^{\prime} 024.279\right)$, Valle del Cauca $\left(2^{\prime} 839.445\right)$, Cundinamarca ( $1^{\prime} 942.377$ y $\left.2^{\prime} 445.101\right)$ y Santander $\left(1^{\prime} 632.263\right.$ y l'381.363). Las entidades territoriales con menor número de dosis asignadas y aplicadas para la vacunación son: Vaupés (20.331 y 17.113), Vichada (26.284 y 22.171), Guainía (28.442 y 22.732), Guaviare (44,840 y 35.919), y Amazonas (76.056 y 67.709). En la figura 2 podemos considerar los porcentajes de dosis asignadas para la vacunación contra SARS-COV-2 en las entidades territoriales de Colombia a 31 de julio 2021. Se puede concluir que aquellas con mayor número de dosis asignadas son: Bogotá (18.61), Antioquia $(14,92)$, Valle del Cauca $(8,82)$, Cundinamarca $(6,03)$, Santander $(5, .07)$ y las entidades territoriales con menor número de porcentaje de dosis asignadas son: Vaupés $(0,66)$, Vichada $(0,08)$, Guaviare $(6.296)$, Guainía $(0,09)$ y Guaviare $(0,14)$. 


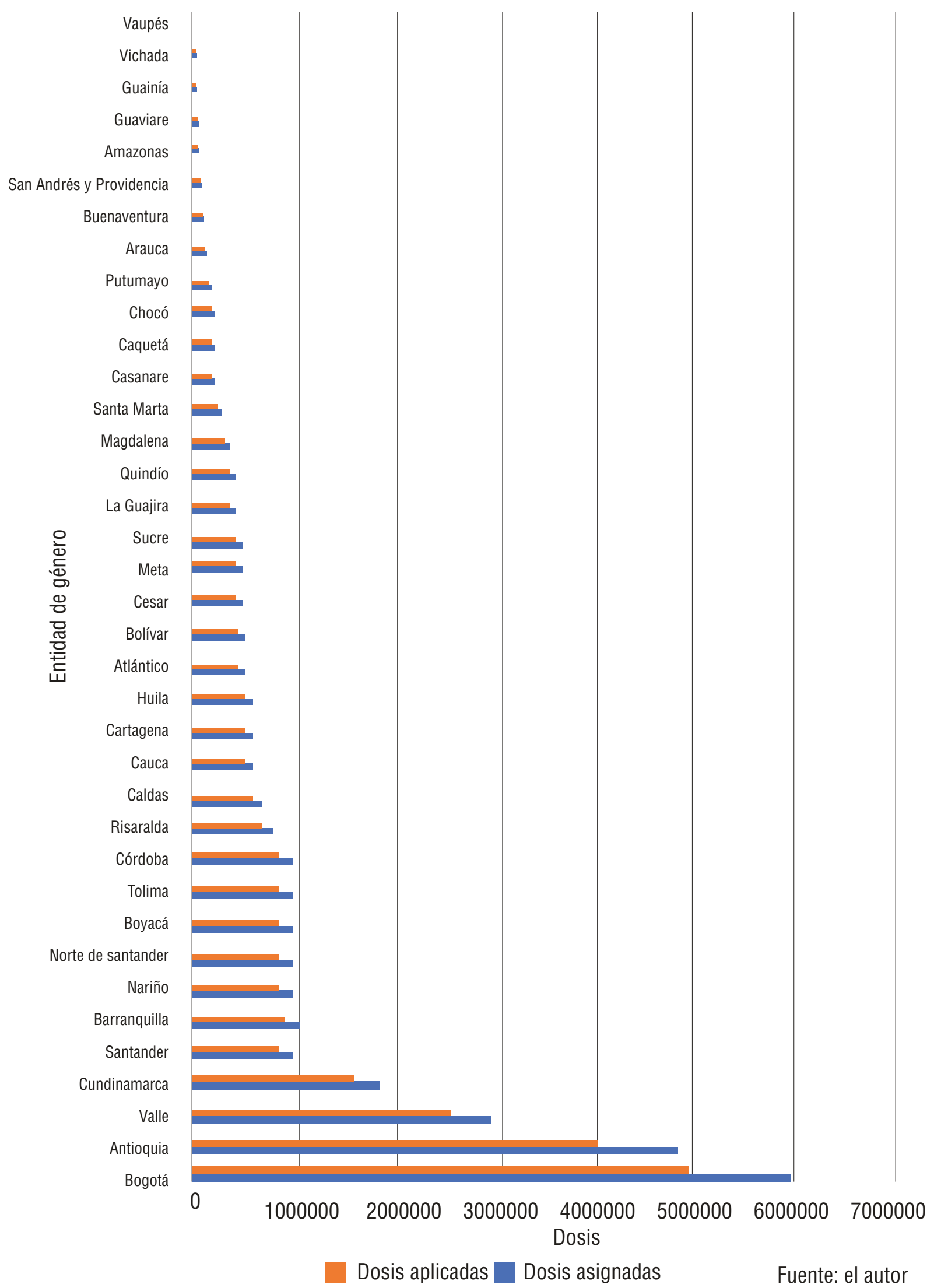

Figura 1. Dosis asignadas y aplicadas por entidad territorial. 


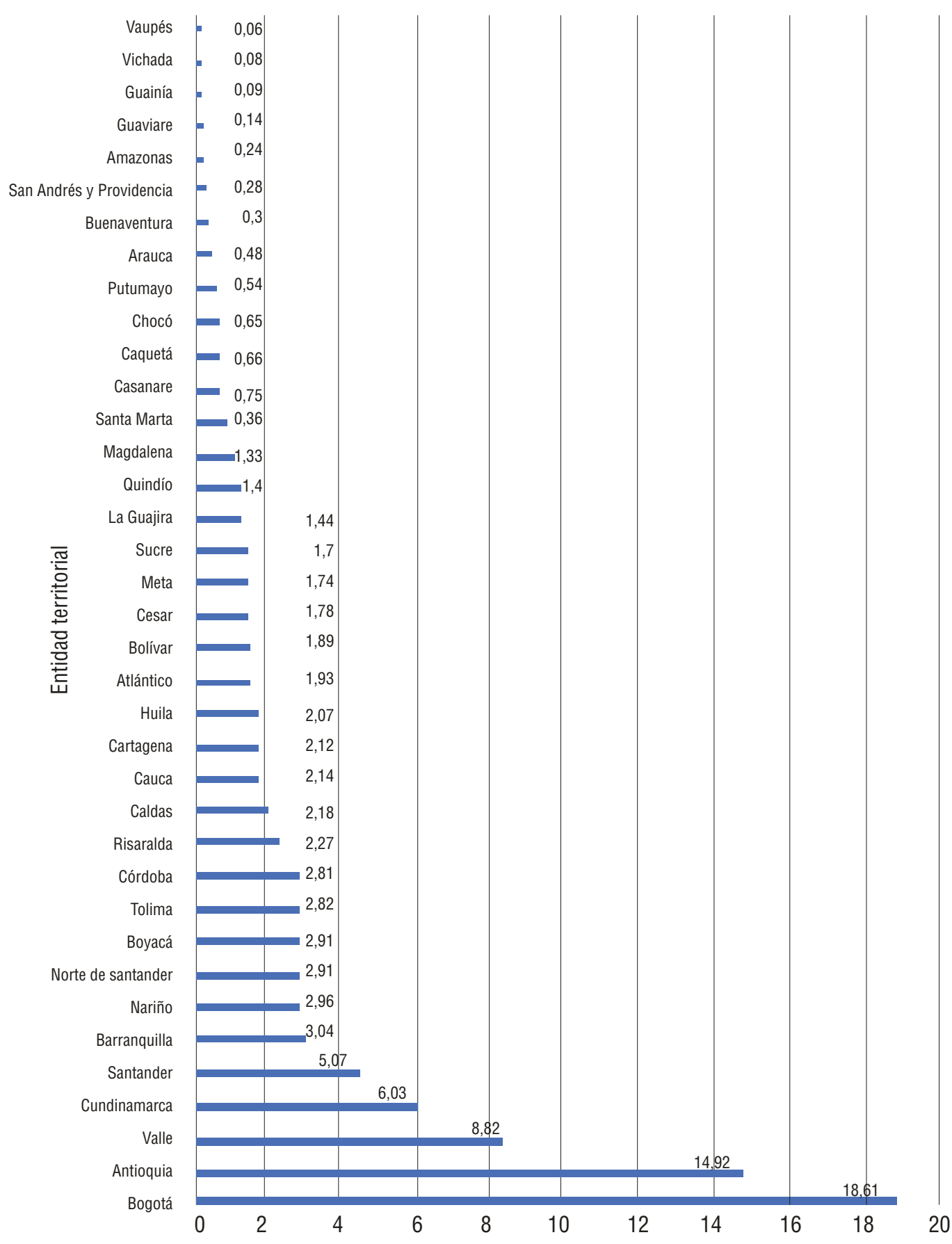

Porcentaje de dosis asignadas

Fuente: el autor

Figura 2. Porcentaje de dosis asignadas por entidad territorial.

En la figura 3 podemos observar los porcentajes de dosis aplicadas en la vacunación contra SARS-COV-2 en las entidades territoriales de Colombia a 31 de julio 2021. Se puede concluir que las entidades territoriales con mayor número de porcentaje de dosis aplicadas de vacunación son: Boyacá $(92,85)$, Buenaventura $(92,82)$, Amazonas $(89,03)$, Cundinamarca $(88,22)$, Nariño $(87)$ y las entidades territoriales con menor número de porcentaje dosis aplicadas de vacunación son: Cauca $(65,28)$, Putumayo $(66,66)$, Chocó $(67,46)$, Magdalena $(77,08)$ y Sucre $(77,21)$. 


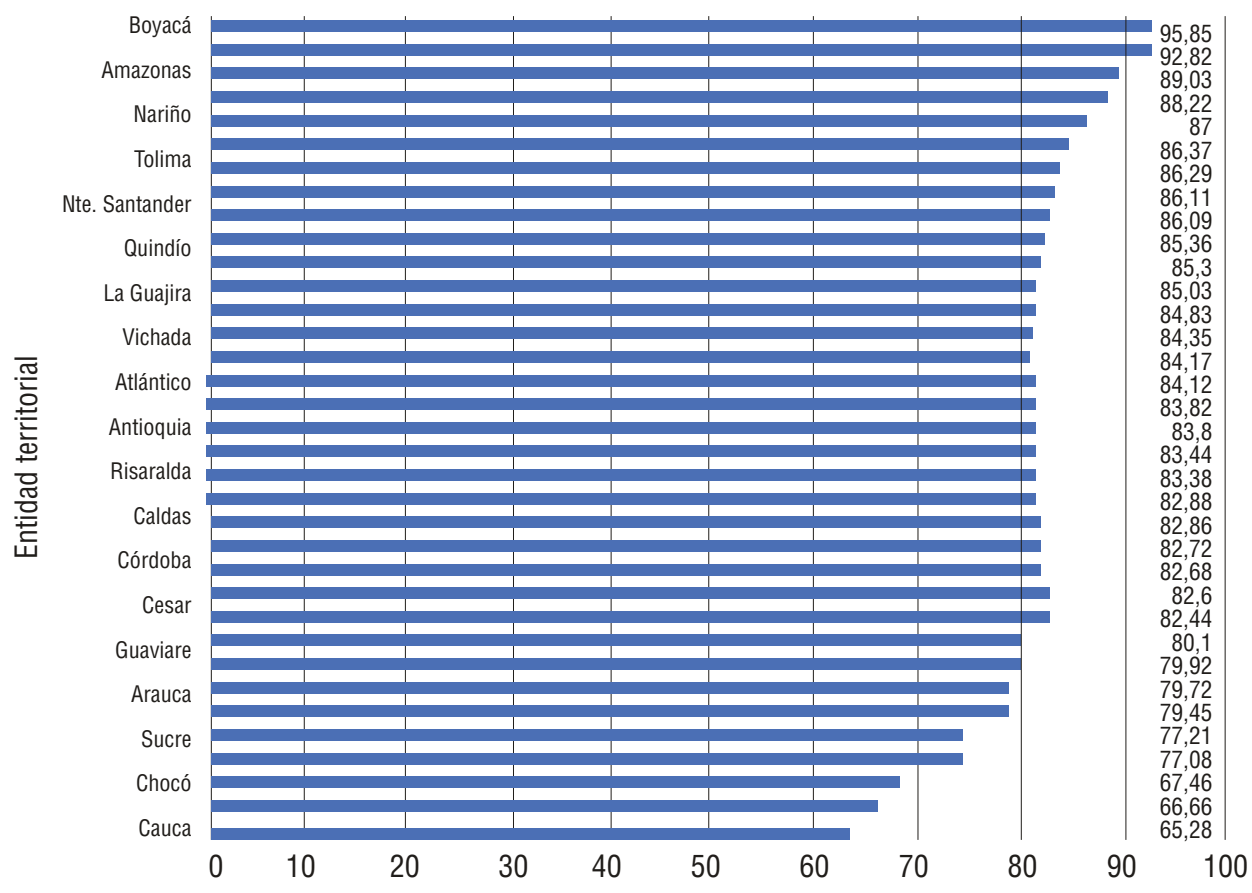

Porcentaje de dosis aplicadas

Fuente: el autor

Figura 3. Porcentaje de dosis aplicadas por entidad territorial.

\section{CONCLUSIONES}

Se estableció que a 31 de julio 2021 las entidades territoriales de Colombia con mayor número de dosis asignadas y aplicadas para la vacunación fueron: Bogotá (5'992.380 y 4'966.730), Antioquia (4'802.193 y 4'024.279), Valle del Cauca (2'839.445), Cundinamarca (1'942.377 y 2'445.101), Santander (1'632.263 y 1'381.363), ahora, las entidades territoriales con mayor número de porcentaje de dosis asignadas fueron: Bogotá (18.61), Antioquia $(14,92)$, Valle del Cauca $(8,82)$, Cundinamarca $(6,03)$, Santander $(5,07)$, y las entidades territoriales con mayor número de porcentaje dosis aplicadas de vacunación fueron: Boyacá $(92,85)$, Buenaventura $(92,82)$, Amazonas $(89,03)$, Cundinamarca $(88,22)$ y Nariño $(87)$. Es relevante continuar aumentado la asignación de dosis contra SARS-COV-2 en los diferentes entes territoriales de Colombia, y a su vez que cada uno aumente su capacidad de aplicaciones y de manera específica en lo relacionado con los esquemas completos, que permitan llegar a corto plazo a alcanzar la inmunidad de rebaño en el país. También es de vital importancia estar muy atentos a las posibles mutaciones del virus, ya que estas pueden repercutir en la inmunidad relacionada por las vacunas, y por lo tanto hay que llevar un proceso continuo de monitoreo en la población y observar si hay algún efecto secundario de la vacuna después de su aplicación.
DECLARACIÓN CONFLICTO DE INTERESES

El autor declara no tener ningún conflicto de intereses.

\section{REFEREN CIAS}

1. Statistics and Research Coronavirus (COVID-19) Vaccinations [Internet]. Our World in Data; 2021 [Consultado 3 de agosto 2021]; Disponible en: https://ourworldindata.org/covid-vaccinations

2. World Health Organization. COVID-19 vaccines [Internet]. World Health Organization; 2021 [Consultado 3 de agosto 2021]. Disponible en: https://www.who.int/emergencies/diseases/novel-coronavirus-2019/covid19-vaccines

3. World Health Organization. COVID-19 vaccine tracker and landscape [Internet]. World Health Organization; 2021 [consultado 3 ago 2021]; Disponible en: https://www.who.int/publications/m/item/draftlandscape-of-covid-19-candidate-vaccines

4. Ramírez, JA. Vacunas para COVID-19. Respirar. 2020;12(1):3-5

5. Center for Disease Control and Prevntion. Science Brief: Emerging SARS-CoV-2 Variants [Internet]. Center for Disease Control and Prevntion; 2021 Consultado 3 agosto 2021 Disponible en: https://www.cdc.gov/coronavirus/2019ncov/science/science-briefs/scientific-brief-emerging-variants.html

6. Kostoff RN, Briggs MB, Porter AL, Spandidos DA, Tsatsakis A. [Comment] COVID-19 vaccine safety. Int J Mol Med. 2020;46(5):1599-1602. doi: 10.3892/ijmm.2020.4733 
7. Krause PR, Gruber MF. Emergency Use Authorization of Covid Vaccines - Safety and Efficacy Follow-up Considerations. N Engl J Med. 2020;383(19):e107. doi: 10.1056/NEJMp2031373

8. Anywaine Z, Whitworth H, Kaleebu P, Praygod G, Shukarev G, et al. Safety and Immunogenicity of a 2-Dose Heterologous Vaccination Regimen With Ad26.ZEBOV and MVA-BN-Filo Ebola Vac: 12-Month Data From a Phase 1 Randomized Clinical Trial in Uganda and Tanzania. J Infect Dis. 2019;220(1):46-56. doi: 10.1093/infdis/jiz070

9. Puthumana J, Egilman AC, Zhang AD, Schwartz JL, Ross JS. Speed, Evidence, and Safety Characteristics of Vaccine Approvals by the US Food and Drug Adminis $\neg$ tration. JAMA Intern Med. 2020;181(4):559560. doi: 10.1001/jamainternmed.2020.7472

10. Spinola SM, Zimet GD, Ott MA, Katz BP. Human Challenge Studies Are Un-likely to Accelerate Cononavirus Vaccine Licensure Due to Ethical and Practical Issues. J Infect Dis. 2020;222(9):1572-1574. doi: 10.1093/infdis/jiaa457

11. Cataño J, Aguirre HD. Vacunación del adulto en tiempos de COVID-19. Corporación de Investigaciones Biológicas. 2020;1(2):1-8.
12. Ministerio de Salud y Protección Social de Colombia. Plan de vacunación nacional contra COVID-19 [Internet]. Ministerio de Salud y Protección Social de Colombia; 2021 [Consultado 3 ago 2021]. Disponible en: https:// www.minsalud.gov.co/salud/publica/Vacunacion/Paginas/Vacunacioncovid-19.aspx

13. Díaz Pinzón JE. Estimación de la prevalencia del COVID-19 en Colombia. Repert Med Cir. 2020;29(Núm. Supl.1):99-102. https://doi.org/10.31260/ RepertMedCir.01217372.1115

14. DíazPinzónJE. Análisis delosresultados del contagio delCOVID-19respecto a su distribución geográfica en Colombia. Repert Med Cir. 2020;29(Núm. Supl.1):60-64. https://doi.org/10.31260/RepertMedCir.01217372.1082

15. Díaz Pinzón, J. E. (2021). Dinámica y relación del contagio del COVID-19 después de iniciado el plan de vacunación contra el SARS-COV-2 en Colombia. Repert Med Cir. 2021;30(Núm. Supl.1):41-45. https://doi. org/10.31260/RepertMedCir.01217372.1227 